\title{
Enhancing Early Engagement (E3) in Mental Health Services Training for Children's Advocacy Center's Victim Advocates: Protocol for a Randomized Clinical Trial
}

Erin K Taylor ( $\square$ erin-taylor@ouhsc.edu )

University of Oklahoma Health Sciences Center https://orcid.org/0000-0001-5581-6611

Alex R Dopp

RAND Corp

Kaitlin Lounsbury

National Children's Alliance

Yutian Thompson

University of Oklahoma Health Sciences Center

Michelle Miller

National Children's Alliance

Ashley G. Jorgensen

University of Oklahoma Health Sciences Center Jane F Silovsky

University of Oklahoma Health Sciences Center

Study protocol

Keywords: child maltreatment, service navigation, Children's Advocacy Centers, training, consultation

Posted Date: August 5th, 2020

DOI: https://doi.org/10.21203/rs.3.rs-51847/v1

License: () (7) This work is licensed under a Creative Commons Attribution 4.0 International License. Read Full License 


\section{Abstract}

Background: Child maltreatment is a major public issue in the United States, yet most children affected by abuse or neglect never engage in evidence-based practices (EBP) for child mental health. Children's Advocacy Centers (CACs') are uniquely situated to serve as Family Navigators who connect children impacted by maltreatment to appropriate EBPs. In fact, the CAC position of Victim Advocate mirrors the Mental Health Family Navigator national initiative.

Methods: We will develop, implement, and evaluate web-based and consultative training for Victim Advocates to enhance early engagement in services (E3 training). The interactive web-based training will embed key targets of knowledge and skills related to family engagement, trauma, and EBP services. Participating CACs will be randomized to E3 webinar-based training, E3 webinar plus consultation, or delayed training. The project will test the E3 training's impact on key mechanisms of change (e.g., knowledge, skills) to improve rates of screening, referral, and access to EBP services. The feasibility of implementing the training program, and differential impact and costs by level of training will be examined.

Discussion: The overarching goal of this project is to test a training that is readily implemented through CACs and examine the mechanisms for improving early engagement and, ultimately, child and adolescent mental health outcomes. Results and cost findings will be used to plan a large-scale comprehensive, mixed-methods Hybrid Type II effectiveness-implementation and cost-effectiveness trial of Family Navigator E3 training. If outcomes are positive, considerable infrastructure exists to support the scale-up and sustainability of E3 training nationwide, by embedding the training in national CAC training protocols.

Trial registration: NCT04221633

\section{Contributions To The Literature}

- Children affected by child abuse and neglect are at high risk for psychological sequela and yet rarely receive evidence-based mental health treatments, even when such practices are available.

- Few training and implementation strategies have been developed to address these challenges.

- The Enhance Early Engagement (E3) training is designed to improve Children's Advocacy Center's Victim Advocate's knowledge and skills at screening for mental health concerns, referral, and engagement in evidence-based practices.

- This protocol describes a randomized pilot trial of feasibility, implementation outcomes (knowledge and skills), mechanisms of effect, and costs across three conditions.

- Results will advance the science behind implementation strategies designed to increase engagement in evidence-based practices, and will help prioritize strategies for additional testing and eventual scale-up.

\section{Background}

Child maltreatment is a major public health issue in the United States (U.S.), with nearly 680,000 children and adolescents confirmed as victims of abuse and neglect in 2018 [1]. The effects of maltreatment and trauma 
on youth are wide-ranging, with both immediate and long-term impacts, including posttraumatic stress disorders [2], disruptive behavior conditions [3, 4], depression [5], and problematic sexual behavior [6]. These problems can persist through adolescence into adulthood and lead to negative well-being, educational, and occupational outcomes, with an annual economic burden reaching $\$ 120$ billion $[7,8]$. A variety of evidence based practices (EBP) have been found to be more efficacious than treatment-as-usual in reducing symptoms for mental health conditions associated with child maltreatment [9]. Unfortunately, the ability of EBP to mitigate the impact of child maltreatment is encumbered because most children in need never engage in EBP [10-15]. In fact, families in most need for intervention have barriers such that they have the most difficulties to engage successfully [15].

We could transform this current state of affairs by capitalizing on the national network of Children's Advocacy Centers (CACs). CACs were created in the mid-1980s to provide coordinated responses to child maltreatment by integrating social, legal, medical, and mental health responses and interventions for child abuse and neglect [16]. Notably, over 370,000 children were served at 900 CACs nationwide in 2019 [17]. Victim Advocates at CACs play a critical role for children, as they are tasked with guiding the family through the entire CAC process, including facilitating access to EBP. National data suggests that Victim Advocates already refer children seen at CACs to mental health services at high rates [18]. Unfortunately, approximately $40 \%$ of those families who receive a mental health referral from CACs never attended an initial appointment [18].

The National Children's Alliance (NCA) is the membership organization and accrediting body for CACs. Through nationwide initiatives over the past decade, NCA has prioritized efforts to improve engagement of children and caregivers served by CACs in mental health screening and EBP treatment [19]. EBP may be provided directly at the CAC or via linkages to providers at community agencies. Victim Advocates are in a prime position to facilitate early engagement in EBP to improve child mental health outcomes. Unfortunately, it is not yet common practice for all Victim Advocates to receive systematic training in mental health screening or engaging children and families in EBP, resulting in significant missed opportunities to streamline families' access to high-quality mental health care. Previous research has found that system navigators with expertise in mental health care are able to connect individuals to evidence-based services successfully and help to maintain their engagement through successful completion of services [20,21]. As such, the next step needed is examining whether the application of training in system navigator knowledge and skills for Victim Advocates will improve family's engagement in EBP.

To evaluate the impact of training, the proposed project will develop, implement, and evaluate the feasibility of a two-level, evidence-informed protocol to train Victim Advocates in (a) mental health screening, (b) family engagement strategies, and (c) EBP identification and referral. The Victim Advocate Enhancing Early Engagement training (E3 training) will seek to improve the short-term mental health outcomes of children affected by maltreatment by improving rates of mental health screening and EBP engagement.

Mental Health Screening. There is no existing structured CAC protocol for Victim Advocates to identify and refer children for mental health services. Furthermore, Victim Advocates are generally bachelor's level professionals who do not have formal training in mental health diagnoses and treatment. Previous research has found that child welfare workers with limited previous training on child mental health and found that 
with brief training they successfully screened over 17,000 children in foster care, with subsequent referrals made to services as needed [22]. Additionally, the only existing study on mental health screeners within CACs found that Victim Advocates reported greater rates of confidence in mental health referral decisions when using a mental health screener [23]. We propose that the E3 training will improve Victim Advocates' identification of children's mental health needs by implementing a consistent process for screening.

Family Engaggement Strategies. Even when Victim Advocates are able to screen and identify the need for child mental health services, they face multiple challenges in successfully linking families to EBP. Low engagement rates of families in mental health treatment are common $[23,24]$. Research has suggested that less than half of Medicaid-eligible families in urban outpatient settings attend an initial appointment [24] and over two-thirds drop out within 7 sessions [25]. Engagement of families in interventions for child maltreatment is wrought with challenges, as families face numerous barriers, including competing demands, stigma associated with mental health services, discrimination, social reactance to court-ordered treatment, and other factors that impact disparities and engagement [24, 27-29]. Thus, engagement is complex, involving individual, familial, provider, agency, and community factors [30]. What appears to make a difference in engagement, despite these challenges, is targeting caregiver perceptions of mental health treatment, while implementing strategies to reduce barriers and promote access, educating about EBP, and providing goal-setting [30]. To this end, E3 training will target these areas by teaching Motivational Interviewing (MI) [31] and the Training Intervention for the Engagement of Families program (TIES) skills [20].

Both $\mathrm{MI}$ and TIES target barriers to engagement in mental health services. MI focuses on addressing a participants' willingness to change and improving intrinsic motivation. Originally developed to address substance abuse, $\mathrm{Ml}$ has been applied to behavioral change in multiple service sectors. Relatedly, TIES addresses perceptual, historical, and external barriers that families face to engaging in mental health services through listening and relationship building skills. TIES embeds $\mathrm{MI}$ in the approach, and both focus on collaborative, empathetic interactions with families from the initial encounter. Both MI and TIES have demonstrated marked increase initial and long-term engagement in mental health services [32, 33].

EBP Identification and Referral. Previous research has demonstrated that combining engagement strategies and EBP leads to significantly improved retention in services and positive outcomes in families involved in child welfare [34-36]. Availability of EBP has increased over the past several decades [37], but they are not yet the norm for mental health services in most communities [38]. Further, it can be challenging for those not trained in mental health to understand what qualifies as EBP and how to identify EBP providers in their own communities. Without a consistently applied protocol for identifying and engaging families in EBPs, children seen through CACs nationwide are less likely to receive needed EBP. To this end, we propose that E3 Training on child mental health, EBP for targeted needs, and identifying EBP in their community will improve families' linkage to EBP by providing Victim Advocates with an evidence-informed approach to identifying and engaging families in services that are most likely to benefit them.

\section{Overview of Study Design}

The three components of the E3 training (i.e., screening, engagement, and referral to EBP) target Victim Advocates' skills and knowledge, improving their efficacy in engaging families in mental health services, 
while also increasing the likelihood that children referred through CACs will receive the best available treatments. Therefore, the current project will expand Victim Advocates' activities of screening and referral by supporting engagement messaging by the Multidisciplinary Team (MDT), identifying EBP in the community, and implementing strategies to overcome barriers. Developing these skills in Victim Advocates will thus help children and adolescents with maltreatment histories receive EBP to address the potential impact of their traumatic experiences. If outcomes are positive, considerable infrastructure exists to support the distribution and sustainability of E3 training, as the training can be readily accessed and embedded in CACs across the nation under the guidance of NCA training protocols.

The primary goal of this pilot study is to examine the initial feasibility, outcomes, and comparative costs of the E3 training. Specifically, we will implement and evaluate a two-level approach to the E3 training: E3w, a professionally developed webinar-only training, compared to E3w+c, the webinar training enhanced by 10 consultation calls with experts in mental health and family engagement. Notably, webinars are cost-efficient compared to in-person training, as they primarily require initial expenditures for their creation and incur only limited additional costs for ongoing maintenance and participation. Webinars can also be made broadly available even in remote settings and require only limited disruption to direct service time when compared to time needed for travel and in-person training. Alternatively, consultation models are inherently more expensive, as they require ongoing staff to provide consultation and require consultants and consultees to devote time that could otherwise be utilized for other valued professional activities (e.g., engaging in client meetings). However, consultation allows for directed practice of skills and continued learning by consultees via guided discussions and role-plays. Previous research on EBP adoption has suggested that ongoing consultation and support improve training outcomes [39]. In addition, practice of skills is crucial for behavior change in mental health settings [40]. Finally, telemedicine technology, similar to what will be used for the consultation calls, has successfully been utilized to deliver coaching and direct feedback for mental health and associated professionals at a lower cost than in-person training while also improving outcomes over the initial training alone [41]. Given the various trade-offs between the E3w and E3w+C approaches, it is critical to compare them directly prior to selecting a candidate training strategy for potential national scale-up of E3 training.

\section{Methods/design}

\section{Study design overview}

For this randomized controlled trial, we will test the feasibility, outcomes, and cost of the two levels of E3 training compared to current practices in CACs in a 1:1:1 allocation ratio. CACs will be randomized to the E3w, E3w+c, or a delayed waitlist control condition. Data will be collected directly from training participants pre-training, immediately post-training, and at follow-up. Further, utilizing NCA's standard data systems (including the Outcome Measurement System, OMS outcome data will be collected for caregivers and community stakeholders pre- and post-training. We hypothesize that the E3 training will be readily implemented within the training structure of NCA and that Victim Advocates and CAC Directors will report high levels of satisfaction with the training. More importantly, we hypothesize that $\mathrm{E} 3 \mathrm{w}$ alone will improve Victim Advocates' knowledge, resulting in minor improvement in EBP engagement, while the addition of 
consultation in E3w+c will lead to increased use of engagement skills, thereby resulting in greater improvement in family engagement in EBP (see Fig. 1 for flow diagram). For purposes of the current study, we will examine family engagement via rates of mental health screening, rates of referral to EBP by Victim Advocates, and family attendance at the first session of EBP. Cost data will be collected to support examining cost-effectiveness in future studies.

\section{Webinar development}

Training for both E3w and $\mathrm{E} 3 \mathrm{~W}+\mathrm{C}$ is planned to be provided using the web-based platform and accessed via the Internet. Although webinars themselves are not unique to the training of professionals in mental health or child maltreatment, by using recommended practices for webinars (e.g., pre-work activities, interactive components, provision of follow-up resources [42], we plan to test an interactive and engaging training.

In addition, a web-based training session is planned for CAC administrators and community stakeholders (i.e., MDT members) across both $\mathrm{E} 3$ and $\mathrm{E} 3+\mathrm{w}$ conditions. The goal of this training is to provide education regarding the role of the Victim Advocate, EBP, and strategies MDT members can use to enhance family engagement in EBP.

\section{Consultation plan}

The E3w+c training also involved two separate orientation training calls for Senior Leaders and Victim Advocates that reviewed the responsibilities and structure of the training. This was followed by 10 consultation/coaching web-based meetings, with the requirement that Victim Advocates attend $80 \%$ for successful completion. These web-based meetings are to occur over a 4 months total. The purpose of starting with weekly meetings is to solidify learning from the webinars. Calls are designed to provide opportunities to individualize learning and practice skills related to mental health screening, engagement (TIES and MI strategies) and linkage to EBP. With Victim Advocates from multiple CACs on each call, there will be shared learning, as each participant will have the opportunity to identify barriers encountered and gain feedback on the application skills.

\section{Site recruitment}

Recruitment and selection of CACs took place in fall 2019. CACs completed an application to participate through NCA, with procedures following NCA's established guidelines for the application, proposal evaluation, site selection, and implementation of training processes. Applications for training were released via email to the accredited CACs across the U.S. Informational calls were held in the fall of 2019 to address questions and review the commitment required for participation in all aspects of the project (training and research). Applicants were reviewed for meeting the following inclusion criteria: (a) Fully accredited by NCA, (b) either directly provides EBP for child mental health or has established and demonstrated linkages for services in the community, (c) participates in the NCA's Outcome Measurement System (OMS), and (d) has Memorandum of Understanding (MOU) or data sharing agreements with all referral sources. Selection was made at the CAC site level, rather than Victim Advocate level. This was to ensure that all Victim Advocates at the same CAC were placed in identical conditions, thereby avoiding any cross-sharing of knowledge across training conditions. See Fig. 2 for a flow-chart regarding enrollment. 


\section{Procedures}

\section{Timeline}

CAC administrators (Senior Leaders) and Victim Advocates from the sites that met the inclusion criteria were invited to participate and complete consenting procedures, as approved by the University of Oklahoma Health Sciences Center. Informed consent was completed with all individual participants via an electronic platform (i.e., REDCap). Participants were informed that they are allowed to discontinue participation as a site or as an individual at any time. Multiple data collection methods are planned for pretraining, post training and follow up (see Fig. 3).. After each webinar, a short training evaluation form is to be sent to E3w and E3w+c participants. Data collection will be monitored by the project coordinator, who will assist sites with any questions or concerns with assistance from the research team. Upon completion of the study, sites will receive a $\$ 600$ payment for their participation.

\section{Data sources}

\section{The Outcome Measurement System (OMS)}

Measures completed by caregivers and MDT members will be captured through the NCA Outcome Measurement System's Initial Visit Caregiver Survey (offered at the end of a CAC visit), Caregiver Follow-Up Survey, and MDT survey. Over the course of the project year, CACs are required to participate in all three OMS surveys. OMS surveys are anonymous and voluntary for caregiver and team member participants and can be delivered in multiple methods, including paper and electronic methods available both on-site and through take-home options. On all three surveys, the questions are a mix of Likert-scale, yes/no, and open-ended items to provide a variety of ways for respondents to share opinions, concerns, and suggestions. The standard OMS surveys were modified to include questions assessing Victim Advocate family engagement skills and connection to EBP.

\section{NCA Member Statistics \& Census}

CACs provide administrative data to NCA on the scope of services provided and remaining service needs through two statistical sources: NCA statistics submitted every 6 months through the NCATrak case management system and NCA Member Census Surveys collected every two years through Qualtrics. Statistics include basic outputs like number of children served, client demographics, and case resolutions. The Census Survey includes more detailed questions on topics like funding sources, staffing information, and information on mental health services provided by CACs and partner agencies. The most recently available Census was collected in the summer of 2018 and the next Census will be distributed in the summer of 2020 .

REDCap

All project-specific data, including measures noted below, client tracking information, and any other assessments completed by the Victim Advocates and Senior Leaders will be collected via REDCap at the University of Oklahoma Health Sciences Center $[43,44]$. Victim Advocates at each site will be provided with a 
user login for REDCap and will be able to enter in data for their site at any time. Advocates will only be able to view the data for their site.

\section{Pre-RCT Survey}

In the first year of the project, we created and implemented an electronic survey of Victim Advocates and Senior Leaders across all CACs. The survey was collected through NCA's Qualtrics system and distributed to the national network of CACs. Survey questions focused on the current roles, responsibilities, activities, tools, and management of Victim Advocates. We received responses from 915 Victim Advocates and 540 CAC Directors. Responses on the survey were utilized by the training team to develop the E3 training. In addition, several items focusing on the mental health screening procedures and barriers that Victim Advocates face when engaging families in EBP were used in the adaptive randomization procedure (see below).

\section{Measures}

Multiple measures will be collected over the course of the study. Details regarding these measures are described in Table 1.

\section{Proposed Mechanisms of Change.}

The key mechanisms proposed for the impact of the E3 training on rates of child mental health screening, referral, and linkage to EBP are changes in Victim Advocates' knowledge and family engagement skills.

A self-report knowledge test will directly examine the knowledge Victim Advocates gain through the training process. Items developed focus on engagement strategies, trauma and effects of trauma, evidence-based mental health treatments, screening for child mental health concerns, and strategies for identifying EBP in their own communities. Our goal is to test change in knowledge acquisition by Victim Advocates.

Skill (i.e., fidelity) measures were adapted from previous research examining self-reported fidelity to the TIES model [45], as well as current coding manuals for MI fidelity $[46,47]$ to create a self-report checklist of skills taught in the training. In consultation with the TIES experts, we developed a self-report checklist that includes both engagement-consistent behaviors (e.g., inquiring about previous mental health experiences) as well as behaviors counter to the MI and TIES strategies (e.g., providing advice). The inclusion of both types of items will ideally decrease the demand for an overly positive response by Victim Advocates.

Factors that can affect the acquisition of knowledge and application of skills can occur at the individual and system level. As such, we included measures of Victim Advocate learning anxiety, motivation, executive functioning, attitudes, and cultural sensitivity, as well as organizational and supervisory culture and support.

\section{Outcome Data}

Targeted outcomes are as follows: implementation of screening, referrals for services, successful linkage to at least one appointment, types of services accessed (i.e., EBP status), and reduced caregiver stress. These are captured via OMS caregiver surveys and through REDCap surveys completed by the Victim Advocate to 
address (a) screening forms implemented, (b) engagement strategies used, (c) results of screening, (d) referrals made, and (e) first treatment session documented by date.

\section{Costs}

To capture direct and indirect costs associated with implementing the E3 training, during the project we will track (a) the amount of time Victim Advocates spend completing the webinar and pre-work activities; (b) the number and length of consultation calls attended by each Victim Advocate (if applicable); and (c) number of screening assessments and referrals completed at each CAC. Detailed cost information will be collected at the follow-up, comprising quantitative and qualitative questions about costs associated with Victim Advocates' activities since completion of the training. Quantitative items are expected to include salary/wages and benefits, time, and resource use, but these items may be modified or supplemented based on qualitative data collected during the training year. The qualitative items will focus on the impact of the family navigator model (e.g., "Describe the impact, if any, that adopting the family navigator role has had on your other job responsibilities”, "What resources will be required to sustain the family navigator role at your CAC?"). Costs associated with development of the training materials and resources will be collected to direct survey of training developers during and post implementation.

\section{Randomization}

A power analysis was conducted initially to determine how many sites would be needed per randomized condition. Because we have three treatment conditions (i.e., E3w, E3w+c, delayed waitlist control), we can assess intervention effects for all three two-way combinations of interventions. The power analyses were conducted for each of these two-way comparisons. To avoid overestimating power [48], we used the smallest number of clusters in an intervention group to estimate power. Power analyses were conducted using the Optimal Design software [49]. With a small intraclass correlation $(\rho=0.05)$ and 50 total CACs (i.e., total clusters across a pair of intervention conditions), the minimal detectable effect size (MDE) is relatively small $\delta=0.19$ as a standardized mean difference assuming $80 \%$ power and a Type I error rate of $5 \%$. This also assumes there are at least 200 referrals per CAC. For the same design criteria and a larger intraclass correlation $(\rho=0.50)$ the MDE is large at 0.57 . Overall, power analyses suggested that we should randomize at least 25 CACs to each condition. We received 114 applications, and the research team evaluated all applications for inclusion criteria. In addition, the research team determined if the CAC lacked capacity to participate in the training (e.g., only one part-time advocate, participation in multiple other training initiatives), they would not be included in the randomization at this time. After review, 81 sites were eligible to participate, and all were randomized.

The analysis process began with a preliminary exploration of baseline covariates that were correlated with the outcome variable caregiver engagement. Variables were taken from pre-existing data collected through the NCA Census $(N=753, q=222)$, NCA statistical data $[N=838, q=68]$ and OMS Surveys (including the caregiver follow-up survey $[N=490, q=16]$, MDT survey $[N=560, q=14]$, CAC director survey $[N=540, q=$ 123], and advocate survey $[N=880, q=156])$. 
Based on factors hypothesized to influence the outcome of interest (i.e., child engagement in EBP), the initial analysis included the followings variables: (a) type of location (urban vs. rural); (b) region of the CAC (e.g., Northeast, Southern), (c) number of children served, (g) number of total CAC staff, (d) number of advocates on staff, (e) organization type (e.g., hospital-based, government based), (f) EBP services provided onsite or via community, (h) level of MDT collaboration, (i) number of barriers CAC staff report experiencing when referring families to EBP, $(j)$ use of a mental health screening tool, $(k)$ advocates previous training experiences, $(I)$ number of children reported to EBP, and $(\mathrm{m})$ number of children who received EBP. The main purpose of the exploratory analysis was to specify the most predictive factors and apply them as the baseline covariates. Both variables $(\mathrm{I})$ and $(\mathrm{m})$ were used as outcomes, and the rest of the variables were predictors in generalized linear models. Because of the exploratory nature of this aim, as well as the existence of missing data, the major risk was a false discovery due to capitalizing on a chance occurrence. Therefore, the analysis practiced the stepwise model selection based on multiple imputed data [50]. Notably, variables (a) region of the CAC, (b) number of barriers when referring families to EBP, and (c) use of a mental health screen tools appeared in more than $50 \%$ of the selected models from twenty imputed data. Therefore, this analysis used these three variables as the covariates in the adaptive randomization.

Covariate adaptive randomization is an approach to ensure that the participants are approximately balanced with respect to covariates in the randomization [51]. The current analysis utilized the method of permuted block randomization with eight stratas (4 region areas $\times 2$ screen tool usage levels) to assign 81 CAC sites randomly into three arms. Group $A(N=26)$, Group $B(N=28)$ and Group $C(N=27)$, corresponding to E3w, $\mathrm{E} 3 \mathrm{~W}+\mathrm{C}$, and Delayed control respectively. A preliminary baseline equivalence test was also applied to check whether any differences between three arms existed. It did not find any difference between groups on children's rate of referral to $\operatorname{EBP}\left(F_{(2,78)}=0.185, p=0.832\right)$, rate of EBP receipt $\left(F_{(2,78)}=0.146, p=0.864\right)$, or number of advocates on $\operatorname{staff}\left(F_{(2,78)}=1.423, p=0.247\right)$.

\section{Proposed Analyses}

For the randomized controlled trial, we will conduct both quantitative and qualitative analyses that will examine the effect of the training on the key outcomes of interest. Proposed analyses are described below.

\section{Quantitative Analytic Plan}

The outcomes analysis will be obtained from the post-training and follow-up assessments of Victim Advocates and Senior Leaders, as well as the continual collection of OMS survey data from caregivers and team members. The variables collected from Victim Advocates and Senior Leaders are the time-varying and CAC-varying provider fidelity, knowledge, and perceptions of the utility of training. The other part of outcomes will relate to family engagement. Statistical analysis will include, but is not limited, to the following: a) applying linear mixed effect models to evaluate the changes of the primary outcomes between conditions across time, should the distribution of the outcomes and residuals suggest being appropriate [52, 53]; and b) investigating the mechanism that is responsible for the causal effect between training conditions and outcomes, with the mediator of knowledge/skill achievement. Covariates collected (e.g., perceived supervisory support, learning anxiety) will also be examined for their influence on the outcome of interest. As a feasibility study, the principal goal in this stage is to examine whether Victim Advocate knowledge and 
skills change due to training, what factors might be associated with the change, and how that influences family engagement in mental health services.

Missing data will not be avoidable due to the large amount of data collected from sites across the national networks, and the repeated measurements across multiple time points. Therefore, stochastic multiple imputation methods will be used to handle missingness, if the assumption of ignorable missing mechanism can be held $[54,55]$. In addition, analyses will be "intent-to-treat," such that individual participants or sites who leave the study will be included in analyses.

All the analysis will be completed by the statistical package R (3.5.2) [56] with multiple packages, such as dplyr, tidyr, ggplot2, Ime4, stats, readr, and mice.

\section{Qualitative Analytic Strategy}

The research team plans to conduct thematic analysis of all qualitative responses on evaluation and followup measures. To do so, all responses to each question will be reviewed in their entirety in order to identify broad themes within the responses. Themes will be organized into a broad codebook, and additional coding will focus on refining themes further. Coding will be conducted by multiple members of the research team, and interrater reliability will be determined through cross-coding of responses and comparison of identified themes. Discrepancies will be reviewed with the larger research team to discuss and finalize coding.

\section{Cost Analysis}

We will generate descriptive statistics from the quantitative cost data to describe typical costs (i.e., means) and variability in costs (i.e., standard deviations) associated with delivery of the E3 training. Direct costs will be calculated in terms of the cost of the resource and the frequency of its use (e.g., consultation fee $x$ number of consultation sessions). Indirect costs will be calculated by applying a shadow price [57], which estimates the value of lost productivity for alternative professional activities of CAC staff, to time spent on training activities (i.e., hourly shadow price $\mathrm{x}$ hours of training activities). All cost estimates will be placed on the same metric through adjustment to (a) an index year using the Consumer Price Index [58] to account for inflation and (b) national average U.S. dollar values using the Council for Community and Economic Research Cost of Living Index [59] to account for costs of living differences between CAC locations. We will sum all direct and indirect expenses separately before calculation of descriptive statistics and examine descriptive statistics for total (i.e., direct plus indirect) costs.

\section{Discussion}

\section{Innovation and anticipated contribution}

Child maltreatment and co-occurring traumas (e.g., domestic violence, parental substance abuse) are core adverse childhood experiences. Mounting research has accumulated demonstrating the immediate and longstanding impact of adverse childhood experiences on physical and mental well-being [60-62]. Effective mitigation of this negative impact involves immediate engagement in EBPs that demonstrate restitution of well-being $[63,64]$. By providing linkages to services upon the outcry of child maltreatment, service 
navigators within CACs can facilitate both the immediate connection to needed mental health treatment as well as address "deep-rooted issues related to distrust in providers and the health system that often lead to avoidance of health problems and non-compliance with treatment recommendations" [65 p. 3543].

The success of previous trials with service navigators within health care settings [65-68] will be built upon for the current feasibility study. Uniquely, the service navigator model tested in this project occurs within the CAC, the location of child forensic interviews and other key interventions provided as part of the investigation of child maltreatment. These multidisciplinary settings are located across the country and connected through the network established by NCA. Nationwide impact on access to EBP for children who experience child maltreatment is feasible given the reach of the 900 CACs across the country [69]. Broadly, no other known existing national system of programs can add one additional component to their existing services (i.e., training Victim Advocates in mental health screening, engagement, and EBP referrals) and have such a widespread impact on the mental health outcomes of children.

The approach of directly addressing disparities in mental health care is another distinction of this trial. Research has suggested that there are significant socioeconomic disparities in the experience of child maltreatment, such that children living in disadvantaged neighborhoods and poverty are disproportionately affected by abuse and neglect [70]. NCA Accreditation Standards, however, require that CACs provide services to clients regardless of their ability to pay, ensuring that all children receive necessary services. In addition, although previous work has suggested that maltreatment may occur at higher rates in minority populations, this effect is largely due to the poverty and marginalization experienced by certain racial groups [71]. As youth who are not White have been found to be less likely to engage in EBP for a variety of reasons, including experiences of discrimination and racism in the service sector $[10,13,14]$, the development and implementation of the E3 training program may help to decrease racial and socioeconomic disparities in the delivery of EBP for children affected by maltreatment. TIES was selected to be a core of E3 training due to its success in enhancing engagement in services by families who are financially disadvantaged and impacted by service disparities $[20,28,24]$.

Victim Advocates within CACs are primed for becoming service navigators given their location, role, and responsibilities. Considering the vulnerabilities of and service disparities experienced by the population served by CACs, successful early engagement in mental health services will need to rise above the baseline of providing education, case management, support and assistance in accessing services. This trial tests the feasibility of integrating well-defined models (i.e., MI and TIES) designed to directly acknowledge and address distrust of service systems and integrate strategies found to reduce internal barriers to change behavior.

Large-scale implementation within complex service systems can be fraught with challenges. Expert recommendation is to construct small changes utilizing the current structure rather than attempt to overhaul the entire system [72]. This logic is readily applied to the current project, as the Victim Advocate position is already embedded and integral to the work of CACs. The E3 training is designed to enhance their capacity, testing whether enhanced skills of the Victim Advocate and the success of early engagement in EBP can be readily accomplished with web-based training alone (E3w) or significantly augmented through consultation $(E 3 w+C)$. The results of this feasibility study will inform a larger randomized trial, integrating longitudinal 
design to examine the impact of Victim Advocate training on the child and family outcomes, as well as potential cost/benefit implications. If successful, the E3 project can inform the development of family navigator models for other settings (e.g., schools) [73] and clinical problems (e.g., problematic sexual behavior, for which service responses are even more challenging to coordinate than for maltreatment) [74].

\section{Limitations/practical \& operational challenges}

Although the current project offers many strengths, limitations and potential challenges warrant comment. Initial plans for data collection were to utilize NCATrak, NCA's proprietary tracking software used by CACs to record data on their clients served, anticipating that most sites would be using the software. However, upon investigation of the potential CAC sites' procedures, the variety of database programs used, and the number of changes required for each system, this was determined to be impractical for collection and integration of data across CACs for the current project. As such, we shifted data collection to one central system, REDCap. However, the decision to use REDCap has not been without challenges, as we have had to train Victim Advocates on the system's procedures and activities. Creating training videos on REDCap procedures and holding open "office hours" with the research team for troubleshooting concerns have facilitated the process.

Further, to examine fidelity to the model, Victim Advocate implementation of engagement strategies like MI and TIES had to be collected via self-report methods, due to the significant impact on the budget of observational methodologies. Notably, research has suggested that professionals are able to reliably selfreport on their use of intervention techniques with a high level of concordance with observer and expert ratings of the same variables $[75,76]$. Observational methodologies are planned to verify changes in skills in the next step of the research.

An unanticipated challenge of the current project has been the rate of job turnover of CAC personnel. In response, we are adding measures related to turnover intent, job satisfaction, and burnout to the post and follow up data collection waves to better understand this phenomenon within the CAC. Our experience highlights the importance of using a readily accessible, effective training platform and the need to examine the impact of training on job retention. Previous research has demonstrated that training in EBP with associated consultation significantly improved job retention of bachelor's level home-based parenting service providers working with vulnerable families (i.e., half the job turnover rate than the other conditions) [77]. Currently, we are implementing an intent-to-train analytic plan by asking those sites who left the training to complete data collection at post-training and follow-up. Lessons learned from this feasibility trial will inform a large randomized trial in the future.

Another unanticipated difficulty has been COVID-19. The pandemic and related stay-at-home orders has had direct and profound impact on the CAC activities as well as the well-being of the staff and the families they serve. Transitioning CAC services to tele-health platforms was essential. Throughout the crisis, we have endeavored to provide support to Victim Advocates in their understanding and skill in administering the screener and using TIES and MI strategies via the phone and telehealth platforms. By providing video training and additional consultation, we hope to broaden the CACs' use of the training. In addition, flexibility and changes in the timeline allowed CACs space to adjust to the changes caused by COVID-19 and improve learning. Finally, measures of COVID-19 impact on well-being and CAC functioning will be integrated in post

Page $13 / 29$ 
and follow up data collection waves, which will provide opportunities to examine the process of family engagement during considerable stress and strain.

\section{Future Directions}

CACs are uniquely well situated to connect families to EBPs in order to target the range of mental health symptoms and disorders of children impacted by maltreatment and other traumatic experiences. The overarching goal of this project is to test the feasibility of a Family Navigator training for Victim Advocates at CACs across the nation and to examine the mechanism that improves children's early engagement in EBP. Ultimately, our goal is to improve child and adolescent mental health outcomes. Quantitative results will allow us to establish the efficacy of the training overall, while qualitative feedback provided by Victim Advocates, CAC directors, and MDT partners will allow us to determine what improvements and changes are needed to the E3 training to allow for wider implementation in the future. Finally, results of the cost analysis will provide critical information about the resources required for E3 training and inform our approach to comparing economic costs and outcomes between different training models. If outcomes are positive, considerable infrastructure exists to support the scale-up and sustainability of E3 training, by embedding the training in all CACs under the guidance of NCA training protocols. Using the results of the current study, we plan to proceed to a larger-scale mixed-methods clinical effectiveness-implementation (Hybrid Type II [78]) and cost-effectiveness trial of the E3 training on child mental health outcomes. These various efforts will support examining broader implementation of the E3 Family Navigator model through CACs nationwide, offering tremendous potential to reduce the social and economic impact of child maltreatment by linking some of our most vulnerable children and families to high-quality mental health treatment.

\section{Declarations}

Ethics approval and consent to participate: Ethics approval was obtained from the Institutional Review Board at the University of Oklahoma Health Sciences Center (Protocol \#11290).

\section{Consent for publication: Not applicable}

Availability of data and materials: The datasets used and/or analyzed during the current study are available from the corresponding author on reasonable request.

Competing interests: The authors declare that they have no competing interests.

Funding. This trial is sponsored by NIMH, research grant R34-MH118486 to the University of Oklahoma (PI: Silovsky). The designated Program Official for the grant is Dr. Denise Pintello (denise.pintello@nih.gov). The funding body and Program Official do not have any role in study design; collection, management, analysis, or interpretation of data; or the writing or submission of this manuscript.

Authors' contributions. ET led the design and execution of this trial under the mentorship of JS, and wrote the first draft of this manuscript. AD assisted with the trial and with writing the first draft of this manuscript, and leads the cost analysis. MM, YT, KL, and AJ assisted with the trial, and reviewed and provided feedback on this manuscript. MM and KL lead the involvement of National Children's Alliance for recruitment and data 
collection. YT leads data management and analysis. AJ coordinates data collection. JS oversees the design and execution of all aspects of this trial as $\mathrm{Pl}$, and oversaw the writing of this manuscript.

Acknowledgements: Not applicable

Authors' information: ${ }^{1}$ Center on Child Abuse and Neglect, Department of Pediatrics, University of Oklahoma Health Sciences Center, 940 NE $13^{\text {th }}$ St \#4900, Oklahoma City, OK 73104; ${ }^{2}$ RAND Corporation, 1776 Main Street, Santa Monica, CA 90401; ${ }^{3}$ National Children's Alliance, 516 C St NE \#100, Washington DC, 20002.

\section{Abbreviations}

CAC: Children's Advocacy Center

E3: enhancing early engagement

E3w: enhancing early engagement training - webinar only

E3w+c: enhancing early engagement training - webinar plus consultation

EBP: evidence-based practice

Ml: motivational interviewing

MDT: Multidisciplinary team

NCA: National Children's Alliance

OMS: Outcome Management System

TIES: Training Intervention for the Engagement of Families

\section{References}

1. U.S. Department of Health \& Human Services, Administration for Children and Families, Administration on Children, Youth and Families, Children's Bureau. Child maltreatment 2015.

http://www.acf.hhs.gov/programs/cb/research-data-technology/statistics-research/child-maltreatment. Accessed 1 March 2020.

2. Jaffee SR. Child maltreatment and risk for psychopathology in childhood and adulthood. Annu Rev Clin Psychol. 2017;13:525-551.

3. Ford JD, Racusin R, Daviss WB, et al. Trauma exposure among children with oppositional defiant disorder and attention deficit-hyperactivity disorder. J Consult Clin Psychol.1999;67(5):786-789.

4. Stouthamer-Loeber M, Loeber R, Homish DL, Wei, E. Maltreatment of boys and the development of disruptive and delinquent behavior. Dev Psychopathol. 2001;13(4):941-955.

5. Toth SL, Manly JT, Cicchetti D. Child maltreatment and vulnerability to depression. Dev 
Psychopathol.1992; 4(1):97-112.

6. Friedrich, WN, Davies W, Feher E, Wright J. Sexual behavior problems in preteen children: Developmental, ecological and behavioral correlates. Ann N Y Acad Sci. 2003;989:95-104.

7. Fang X, Brown DS, Florence CS, Mercy JA. The economic burden of child maltreatment in the United States and implications for prevention. Child Abuse Negl. 2012;36(2):156-165.

8. Gustavsson A, Svensson M, Jacobi F, et al. Cost of disorders of the brain in Europe 2010. Eur Neuropsychopharmacol. 2011;21:718-779.

9. Chorpita, BF, Daleiden, EL, Ebesutani, C., et al. Evidence-based treatments for children and adolescents: An updated review of indicators of efficacy and effectiveness. Clin Psychol Sci Prac. 2011;18:154-172.

10. Burns BJ, Phillips SD, Wagner HR, et al. Mental health need and access to mental health services by youths involved with child welfare: A national survey. J Am Acad Child Adolesc Psychiatry. 2004;43(8):960970.

11. Horwitz SM, Hurlburt MS, Heneghan A, et al. Mental health problems in young children investigated by US child welfare agencies. J Am Acad Child Adolesc Psychiatry. 2012;51(6):572-581.

12. Hurlburt MS, Leslie LK, Landsverk J, et al. Contextual predictors of mental health service use among children open to child welfare. Arch Gen Psychiatry. 2004;61(12):1217-1224.

13. Garcia A, Courtney M. Prevalence and predictors of service utilization among racially and ethnically diverse adolescents in foster care diagnosed with mental health and substance abuse disorders. J Public Child Welf. 2011;5(5):521-545.

14. Stein RE, Hurlburt MS, Heneghan AM, et al. For better or worse? Change in service use by children investigated by child welfare over a decade. Acad Pediatr. 2016;16(3):240-246.

15. Vanderzee KL, Pemberton JR, Conners-Burrow N, Kramer TL. Who is advocating for children under six? Uncovering unmet needs in child advocacy centers. Child Youth Serv Rev. 2016;61:303-310.

16. National Children's Alliance. NCA organizational history and background. Washington, DC: NCA; 2016.

17. National Children's Alliance. NCA national statistics - Final report. Washington, DC: NCA; 2019.

18. National Children's Alliance. Healing, justice, \& trust - A national report on outcomes for Children's Advocacy Centers: Member edition. Washington, DC: NCA; 2019.

19. National Children's Alliance. Thriving Kids 2019: A national report on mental health outcomes in Children's Advocacy Centers. Washington, DC: NCA; 2019.

20. McKay MM, McCadam K, Gonzales J. Addressing the barriers to mental health services for inner city children and their caretakers. Community Ment Health J. 1996;32(4):353-361.

21. McKay MM, Nudelman R, McCadam K, Gonzales J. Evaluating a social work engagement approach to involving inner-city children and their families in mental health care. Res Soc Work Pract.1996;6(4):462-472. 22. Wilsie CC, Hunter M, Wilson K. Oklahoma Trauma Assessment and Service Center Collaborative Final Report. Washington, D.C.: Department of Health and Human Services, Children's Bureau: 2018.

23. Conners-Burrow NA, Tempel AB, Sigel BA, Church, JK, Kramer TL, Worley KB. The development of a systematic approach to mental health screening in Child Advocacy Centers. Child Youth Serv Rev. 2012;34(9):1675-1682.

24. Harrison ME, McKay MM, Bannon WM. Inner-city child mental health service use: The real question is why youth and families do not use services. Community Ment Health J. 2004;40(2):119-131.

25. McKay MM, Harrison ME, Gonzales J, Kim L, Quintana E. Multiple-family groups for urban children with 
conduct difficulties and their families. Psychiatr Serv. 2002;53(11):1467-1468.

26. McKay MM, Stoewe J, McCadam K, Gonzales J. Increasing access to child mental health services for urban children and their caregivers. Health Soc Work.1998;23(1):9-15.

27. Beasley LO, Silovsky JF, Ridings LE, Smith T, Owora AH. Understanding program engagement and attrition in child abuse prevention: A mixed-methods approach. J Family Strengths. 2014;14(1):1-24.

28. Damashek A, Doughty D, Ware L, Silovsky JF. Predictors of treatment engagement and attrition in homebased child maltreatment prevention services. Child Maltreat. 2011;16(1):9-20

29. Dempster R, Winders Davis D, Jones VF, Keating A, Wildman B. The role of stigma in parental helpseeking for perceived child behavior problems in urban, low-income African American parents. J Clin Psychol Med Settings. 2018;22:265-275.

30. Becker KD, Boustani M, Gellatly R, Chorpita BF. Forty years of engagement research in children's mental health services: Multidimensional measurement and practice elements. J Clin Child Adolesc Psychol. 2018;47(1):1-23.

31. Miller WR, Rollnick S. Motivational interviewing: Preparing people for change. New York, NY: Guilford Press; 1991.

32. McKay MM, Harrison ME, Gonzales J, Kim L, Quintana E. Multiple-family groups for urban children with conduct difficulties and their families. Psychiatr Serv. 2002;53(11):1467-1468.

33. Romano M, Peters L. Evaluating the mechanisms of change in motivational interviewing in the treatment of mental health problems: A review and meta-analysis. Clin Psychol Rev. 2015;38:1-12.

34. Chaffin MC, Silovsky JF, Funderburk B, et al. Parent-child interaction therapy with physically abusive parents: Efficacy for reducing future abuse reports. J Consult Clin Psychol. 2004;72(3):500-510.

35. Chaffin M, Valle L, Funderburk B, et al. A motivational intervention can improve retention in PCIT for lowmotivation child welfare clients. Child Maltreat. 2009;14(4):356-368.

36. Chaffin MC, Funderburk B, Bard D, Valle LA, Gurwitch RA. Combined motivation and parent-child interaction therapy package reduces child welfare recidivism in a randomized dismantling field trial. $\mathrm{J}$ Consult Clin Psychol. 2010;79(1):84-95.

37. Cooper JL, Aratani Y. The status of states' policies to support evidence-based practices in children's mental health. Psychiatr Serv. 2009;60(12):1672-1675.

38. Becker EM, Smith AM, Jensen-Doss A. Who's using treatment manuals? A national survey of practicing therapists. Behav Res Ther. 2013;51(10):706-710.

39. Herschell AD, Kolko DJ, Baumann BL, Davis AC. The role of therapist training in the implementation of psychosocial treatments: A review and critique with recommendations. Clin Psychol Rev. 2010;30(4):448466.

40. Bearman SK, Weisz JR, Chorpita BF, et al. More practice, less preach? The role of supervision processes and therapist characteristics in EBP implementation. Admin Policy Ment Health. 2013;40(6):518-529.

41. Funderburk BW, Ware LM, Altshuler E, Chaffin M. Use and feasibility of telemedicine technology in the dissemination of parent-child interaction therapy. Child Maltreat. 2008;13(4):377-382.

42. Zoumenou V, Sigman-Grant M, Coleman G, et al. Identifying best practices for an interactive webinar. J Fam Consum Sci. 2015;107(2):62-69.

43. Harris PA, Taylor R, Thielke R, Payne J, Gonzalez N, Conde JG. Research electronic data capture (REDCap) - A metadata-driven methodology and workflow process for providing translational research informatics 
support. J Biomed Inform. 2009;42(2):377-381.

44. Harris PA, Taylor R, Minor BL, Elliott V, Fernandez M, O'Neal L, et al. The REDCap consortium: Building an international community of software partners. J Biomed Inform. 2019; 95:103208.

45. Dorsey S, Pullmann MD, Berliner L, Koschmann E, McKay M, Deblinger E. Engaging foster parents in treatment: a randomized trial of supplementing trauma-focused cognitive behavioral therapy with evidencebased engagement strategies. Child Abuse Negl. 2014;38(9):1508-1520.

46. Miller WR, Moyers TB, Ernst D, Armhein P. (2008). Manual for the motivational interviewing skill code (MISC). https://casaa.unm.edu/download/misc.pdf. Accessed February 4, 2018.

47. Moyers, TB, Rowell LN, Manuel JK, Ernst D, Houck JM. The motivational interviewing treatment integrity code (MITI 4): rationale, preliminary reliability and validity. J Subst Abuse Treat. 2016;65:36-42.

48. Candel MJ, Van Breukelen GJ. Varying cluster sizes in trials with clusters in one treatment arm: Sample size adjustments when testing treatment effects with linear mixed models. Stat Med. 2009;28(18):23072324.

49. Spybrook J, Raudenbush SW, Congdon R, Martinez A. Optimal design for longitudinal and multilevel research: Documentation for the "optimal design" software. Available from:

http://people.cehd.tamu.edu/ okwok/epsy652/OD/od-manual-20080312-v176.pdf [Accessed 13 February 2018].

50. Wood AM, White IR, Royston P. How should variable selection be performed with multiply imputed data? Statistics in medicine. 2008;27(17):3227-46.

51. US Department of Health and Human Services, Food and Drug Administration, Center for Drug Evaluation and Research, Center for Biologics Evaluation and Research. Adaptive designs for clinical trials of drugs and biologics guidance for industry. [internet]. Washington, DC; US Department of Health and Human Services. 2019 [updated 2019 Dec; cited 2020 July]. Available from: https://www.fda.gov/regulatoryinformation/search-fda-guidance-documents/adaptive-design-clinical-trials-drugs-and-biologics-guidanceindustry.

52. Raudenbush SW, Bryk AS. Hierarchical linear models: Applications and data analysis methods. Sage; 2002.

53. Bates D, Mächler M, Bolker B, Walker S. Fitting linear mixed-effects models using Ime4. arXiv preprint arXiv:1406.5823. 2014 Jun 23.

54. Enders CK. Applied missing data analysis. New York: Guilford Press; 2010.

55. Little RJ, Rubin DB. Statistical analysis with missing data. Hoboken, NJ: Wiley; 2020.

56. R Core Team. R: A language and environment for statistical computing. Vienna, Austria: R Foundation for Statistical Computing. Available from: URL https://www.R-project.org/ [Accessed 15 December 2019].

57. Heckman J. Shadow prices, market wages, and labor supply. Econometrica.1974;42(4): 679-694.

58. Bureau of Labor Statistics. Consumer Price Index inflation calculator. https://data.bls.gov/cgibin/cpicalc.pl [Accessed 10 July 2020].

59. Council for Community and Economic Research. Cost of living index. https://www.c2er.org/ [Accessed 10 July 2020].

60. Dube SR, Anda RF, Whitfield, CL, Brown DW, Felitti VJ, Dong M, et al. Long-term consequences of childhood sexual abuse by gender of victim. Am J Prev Med. 2005;28:430-438.

61. Edwards VJ, Anda RF, Dube SR, Dong M, Chapman DF, Felitti VJ. The wide-ranging health consequences 
of adverse childhood experiences. In Kathleen Kendall-Tackett, Sarah Giacomoni, eds. Victimization of Children and Youth: Patterns of Abuse, Response Strategies. Kingston, NJ: Civic Research Institute; 2005. 62. Merrick MT, Guinn AS. (2018). Child abuse and neglect: Breaking the intergenerational link. J Am Public Health Assoc. 2018;108(9):1117-1118.

63. California Evidence-Based Clearinghouse for Child Welfare. Trauma-focused cognitive-behavioral therapy (TF-CBT). Available from: http://www.cebc4cw.org/program/trauma-focused-cognitive-behavioral-therapy/ [Accessed 15 July 2020].

64. Deblinger E, Mannarino AP, Cohen JA, Steer RA. A multisite, randomized controlled trial for children with sexual abuse related PTSD symptoms. J Am Acad Child Adolesc Psychiatry. 2006;45:1474-1484.

65. Natale-Pereira A, Enard KR, Nevarez L, Jones LA. The role of patient navigators in eliminating health disparities. Cancer. 2011;117(S15):3541-50.

66. Ferrante JM, Cohen DJ, Crosson, JC. Translating the patient navigator approach to meet the needs of primary care. J Am Board Fam Med. 2010;23:736-744.

67. Szilagyi PG, Humiston SG, Gallivan S, Albertin C, Sandler M, Blumkin A. Effectiveness of a citywide patient immunization navigator program on improving adolescent immunizations and preventive care visit rates. Arch Pediatr Adolesc Med. 2011;165(6): 547-553.

68. Van Walleghem N, MacDonald CA, Dean HJ. The Maestro Project: A patient navigator for the transition of care for youth with type 1 diabetes. Diabetes Spectr. 2011;24(1):9-13.

69. National Children's Alliance. CAC Coverage Maps [internet]. Washington, DC: National Children's Alliance; 2019. Available at: https://www.nationalchildrensalliance.org/cac-coverage-maps/ [Accessed 20200722 ].

70. Drake B, Jonson-Reid M. Poverty and child maltreatment. In Korbin J, Krugman R, eds. Handbook of Child Maltreatment. Dordrecht, Netherlands: Springer; 2014(2): 131-148.

71. Putnam-Hornstein E, Needell B, King B, Johnson-Motoyama M. Racial and ethnic disparities: A population-based examination of risk factors for involvement with child protective services. Child Abuse Negl. 2013;37(1):33-46.

72. Aarons GA, Chaffin M. Scaling-up evidence-based practices in child welfare services systems. CYF News. 2013 Apr;5.

73. Brueck M. Promoting access to school-based services for children's mental health. AMA J Ethics. 2016;18:1218-1224.

74. Kelley A, Shawler P, Shields JD, Silovsky JF. A qualitative investigation of policy for youth with problematic sexual behavior. Am J Community Psychol, 2019;47(6):1347-1363.

75. Chapman JE, McCart MR, Letourneau EJ, Sheidow AJ. Comparison of youth, caregiver, therapist, trained, and treatment expert raters of therapist adherence to a substance abuse treatment protocol. $\mathrm{J}$ Consult Clin Psychol. 2013;81(4):674.

76. Hogue A, Dauber S, Henderson CE, Liddle HA. Reliability of therapist self-report on treatment targets and focus in family-based intervention. Adm Policy Ment Health. 2014;41(5):697-705.

77. Aarons GA, Sommerfeld D, Hecht D, Silovsky JF, Chaffin M. The impact of evidence-based practice implementation and fidelity monitoring on staff turnover: Evidence for a protective effect. J Consult Clin Psychol. 2009;77(2):270-280.

78. Curran GM, Bauer M, Mittman B, Pyne JM, Stetler C. Effectiveness-implementation hybrid designs: Combining elements of clinical effectiveness and implementation research to enhance public health impact. 
Med Care. 2012;50(3):217-226.

79. Ho MK. Use of Ethnic Sensitive Inventory (ESI) to enhance practitioner skills with minorities. J Multicult Soc Work. 1991;1:57-67.

80. Eisenberger R, Huntington R, Hutchison S, Sowa D. Perceived organizational support. J Appl Psychol. 1986;17(3):200-507.

81. Eisenberger R, Stinglhamber F, Vandenberghe C, Sucharski IL, Rhoades L. Perceived supervisor support: contributions to perceived organizational support and employee retention. J Appl Psychol. 2002;87(3):565573.

82. Chen G, Gully SM, Eden D. Validation of a new general self-efficacy scale. Organ Res Methods. 2001;4(1):62-83.

83. Roth RM, Isquith PK, Gioia, GA. Behavioral Rating Inventory of Executive Function-Adult version. Lutz, FL: Psychological Assessment Resources, Inc.; 2005.

84. Noe RA., Schmitt N. The influence of trainee attitudes on training effectiveness: a test of a model. Pers Psychol. 1986;39:497-523.

85. Noe RA, Wilk SL. Investigation of the factors that influence employees' participation in development activities. J Appl Psychol. 1993;78(2):291-302.

86. Jellinek MS, Murphy JM, Little M, Pagano ME, Comer DM, Kelleher KJ. Use of the Pediatric Symptom Checklist to screen for psychosocial problems in pediatric primary care: a national feasibility study. Arch Pediatr Adolesc Med. 1999;153(3):254-260.

\section{Table}

Table 1. Assessment measures across participants and time points. 


\begin{tabular}{|c|c|c|c|c|c|}
\hline Construct & Measure & $\begin{array}{l}\text { Brief } \\
\text { Description }\end{array}$ & $\begin{array}{l}\text { Time } \\
\text { points }\end{array}$ & Participants & Method/procedure \\
\hline \multicolumn{6}{|c|}{$\begin{array}{l}\text { Proposed } \\
\text { Mechanisms of } \\
\text { Change }\end{array}$} \\
\hline \multirow[t]{2}{*}{ Knowledge } & $\begin{array}{l}\text { Test of } \\
\text { knowledge }\end{array}$ & $\begin{array}{l}\text { 39-items } \\
\text { developed to } \\
\text { assess } \\
\text { advocates } \\
\text { knowledge of } \\
\text { screening, } \\
\text { barriers, } \\
\text { engagement } \\
\text { strategies, } \\
\text { and trauma; } \\
\text { mixed format } \\
\text { of multiple } \\
\text { choice and } \\
\text { true/false }\end{array}$ & $\begin{array}{l}\text { Pre- } \\
\text { training, } \\
\text { Post- } \\
\text { training, } \\
\text { Follow-up }\end{array}$ & $\begin{array}{l}\text { Victim } \\
\text { Advocate }\end{array}$ & $\begin{array}{l}\text { Self-Report via } \\
\text { REDCap }\end{array}$ \\
\hline & $\begin{array}{l}\text { Self- } \\
\text { Evaluation of } \\
\text { Knowledge }\end{array}$ & $\begin{array}{l}20 \text {-item } \\
\text { measure } \\
\text { assessing } \\
\text { self-perceived } \\
\text { knowledge of } \\
\text { evidence- } \\
\text { based } \\
\text { treatment, } \\
\text { mental health, } \\
\text { screening, } \\
\text { trauma, and } \\
\text { engagement } \\
\text { strategies }\end{array}$ & $\begin{array}{l}\text { Pre- } \\
\text { training, } \\
\text { Post- } \\
\text { training, } \\
\text { Follow-up }\end{array}$ & $\begin{array}{l}\text { Victim } \\
\text { Advocate }\end{array}$ & $\begin{array}{l}\text { Self-Report via } \\
\text { REDCap }\end{array}$ \\
\hline Skills & Fidelity & $\begin{array}{l}\text { Items } \\
\text { developed for } \\
\text { respondents } \\
\text { to rate use of } \\
\text { engagement } \\
\text { skills during } \\
\text { meeting with } \\
\text { caregiver }\end{array}$ & $\begin{array}{l}\text { Pre- } \\
\text { training, } \\
\text { Post- } \\
\text { training, } \\
\text { Follow-up }\end{array}$ & $\begin{array}{l}\text { Victim } \\
\text { Advocate }\end{array}$ & $\begin{array}{l}\text { Self-Report via } \\
\text { REDCap }\end{array}$ \\
\hline \multicolumn{6}{|l|}{$\begin{array}{l}\text { Potential } \\
\text { Moderators }\end{array}$} \\
\hline Attitudes & $\begin{array}{l}\text { Victim } \\
\text { Advocate } \\
\text { Attitudes }\end{array}$ & $\begin{array}{l}\text { 22-items } \\
\text { developed to } \\
\text { assess } \\
\text { respondents' } \\
\text { attitudes } \\
\text { towards } \\
\text { mental health, } \\
\text { screening, }\end{array}$ & $\begin{array}{l}\text { Pre- } \\
\text { training, } \\
\text { Post- } \\
\text { training, } \\
\text { Follow-up }\end{array}$ & $\begin{array}{l}\text { Victim } \\
\text { Advocate }\end{array}$ & $\begin{array}{l}\text { Self-Report via } \\
\text { REDCap }\end{array}$ \\
\hline
\end{tabular}


trauma, and

supervision

\begin{tabular}{|c|c|c|c|c|c|}
\hline $\begin{array}{l}\text { Barriers to } \\
\text { treatment }\end{array}$ & $\begin{array}{l}\text { Perceived } \\
\text { Barriers to } \\
\text { Treatment }\end{array}$ & $\begin{array}{l}\text { Advocates } \\
\text { perceptions of } \\
\text { barriers } \\
\text { families face } \\
\text { when } \\
\text { attempting to } \\
\text { access } \\
\text { evidence- } \\
\text { based mental } \\
\text { health } \\
\text { services }\end{array}$ & $\begin{array}{l}\text { Pre- } \\
\text { training, } \\
\text { Post- } \\
\text { training, } \\
\text { Follow-up }\end{array}$ & $\begin{array}{l}\text { Victim } \\
\text { Advocate }\end{array}$ & $\begin{array}{l}\text { Self-Report via } \\
\text { REDCap }\end{array}$ \\
\hline
\end{tabular}

$\begin{array}{clllll}\text { Ethnic } & \text { Ethnic } & \text { 5-items } & \text { Pre- } & \text { Victim } & \text { Self-Report via } \\ \text { Sensitivity } & \text { Sensitivity } & \text { assessing } & \text { training, } & \text { Advocate } & \text { REDCap } \\ & \text { Inventory: } & \text { respondent's } & \text { Post- } & & \\ & \text { Precontact } & \text { sensitivity to } & \text { training, } & \\ & \text { [79] } & \text { other cultures } & \text { Follow-up } & \\ & \text { and } & & \\ & & & & \end{array}$

Organizational

support
16 items

assessing

respondents'

perceptions of

the support

they receive

from their

agency
Pre- Victim Self-Report via

training, Advocate REDCap

Post-

training,

Follow-up
Supervisory

support

Perceived
Supervisory
support [81]

16 items

assessing respondents' perceptions of the support they receive from their supervisor

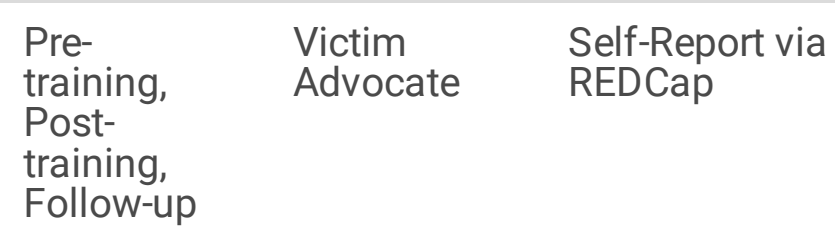

Pre- Victim Self-Report via training, Advocate REDCap Posttraining, Follow-up 


\begin{tabular}{|c|c|c|c|c|c|}
\hline $\begin{array}{l}\text { Executive } \\
\text { functioning }\end{array}$ & $\begin{array}{l}\text { Behavior } \\
\text { Rating } \\
\text { Inventory of } \\
\text { Executive } \\
\text { Function-Adult } \\
\text { [83] }\end{array}$ & $\begin{array}{l}\text { Assessment } \\
\text { of executive } \\
\text { functioning } \\
\text { abilities } \\
\text { (abbreviated } \\
\text { version) }\end{array}$ & Pre-training & $\begin{array}{l}\text { Victim } \\
\text { Advocate }\end{array}$ & $\begin{array}{l}\text { Self-Report via } \\
\text { REDCap }\end{array}$ \\
\hline $\begin{array}{l}\text { Learning } \\
\text { anxiety }\end{array}$ & $\begin{array}{l}\text { Learner } \\
\text { Anxiety } \\
\text { Assessment }\end{array}$ & $\begin{array}{l}\text { 3-item } \\
\text { measure } \\
\text { assessing } \\
\text { concerns } \\
\text { regarding } \\
\text { learning new } \\
\text { information }\end{array}$ & Pre-training & $\begin{array}{l}\text { Victim } \\
\text { Advocate }\end{array}$ & $\begin{array}{l}\text { Self-Report via } \\
\text { REDCap }\end{array}$ \\
\hline \multirow[t]{2}{*}{$\begin{array}{l}\text { Motivation to } \\
\text { Learn }\end{array}$} & $\begin{array}{l}\text { Motivation to } \\
\text { Learn - } \\
\text { General } \\
\text { [adapted from } \\
84,85]\end{array}$ & $\begin{array}{l}3 \text { items } \\
\text { assessing } \\
\text { trait-like } \\
\text { motivation to } \\
\text { learn new } \\
\text { things }\end{array}$ & Pre-training & $\begin{array}{l}\text { Victim } \\
\text { Advocate }\end{array}$ & $\begin{array}{l}\text { Self-Report via } \\
\text { REDCap }\end{array}$ \\
\hline & $\begin{array}{l}\text { Motivation to } \\
\text { Learn - } \\
\text { Specific } \\
\text { [adapted from } \\
84,85]\end{array}$ & $\begin{array}{l}\text { 5-items } \\
\text { assessing } \\
\text { motivation to } \\
\text { learn } \\
\text { information } \\
\text { specific to the } \\
\text { E3 training }\end{array}$ & Pre-training & $\begin{array}{l}\text { Victim } \\
\text { Advocate }\end{array}$ & $\begin{array}{l}\text { Self-Report via } \\
\text { REDCap }\end{array}$ \\
\hline \multicolumn{6}{|l|}{$\begin{array}{l}\text { Training } \\
\text { Evaluation }\end{array}$} \\
\hline $\begin{array}{l}\text { Webinar } \\
\text { evaluation }\end{array}$ & $\begin{array}{l}\text { Post-webinar } \\
\text { evaluation }\end{array}$ & $\begin{array}{l}\text { Brief measure } \\
\text { assessing } \\
\text { satisfaction } \\
\text { with each } \\
\text { webinar and } \\
\text { feedback } \\
\text { regarding } \\
\text { potential } \\
\text { improvements }\end{array}$ & $\begin{array}{l}\text { Completed } \\
\text { after each } \\
\text { webinar }\end{array}$ & $\begin{array}{l}\text { Victim } \\
\text { Advocate }\end{array}$ & $\begin{array}{l}\text { Self-Report via } \\
\text { REDCap }\end{array}$ \\
\hline \multirow{2}{*}{$\begin{array}{l}\text { Overall } \\
\text { training } \\
\text { evaluation }\end{array}$} & \multirow[t]{2}{*}{$\begin{array}{l}\text { Post-training } \\
\text { evaluation }\end{array}$} & \multirow{2}{*}{$\begin{array}{l}\text { Measure } \\
\text { assessing } \\
\text { overall } \\
\text { satisfaction } \\
\text { and feedback } \\
\text { regarding E3 } \\
\text { training, } \\
\text { including } \\
\text { webinars, } \\
\text { consult calls } \\
\text { (when } \\
\text { applicable), }\end{array}$} & \multirow[t]{2}{*}{$\begin{array}{l}\text { Post- } \\
\text { training }\end{array}$} & $\begin{array}{l}\text { Victim } \\
\text { Advocate }\end{array}$ & \multirow[t]{2}{*}{$\begin{array}{l}\text { Self-Report via } \\
\text { REDCap }\end{array}$} \\
\hline & & & & $\begin{array}{l}\text { Senior } \\
\text { Leader }\end{array}$ & \\
\hline
\end{tabular}


Participant and

Agency

characteristics

\begin{tabular}{clllll} 
Demographics & $\begin{array}{l}\text { Demographic } \\
\text { Questionnaire }\end{array}$ & $\begin{array}{l}\text { Basic } \\
\text { demographic } \\
\text { information }\end{array}$ & $\begin{array}{l}\text { Pre- } \\
\text { training, } \\
\text { Post- } \\
\text { training, }\end{array}$ & $\begin{array}{l}\text { Victim } \\
\text { Advocate }\end{array}$ & $\begin{array}{l}\text { Self-Report via } \\
\text { REDCap }\end{array}$ \\
\cline { 5 - 6 } & & Follow-up & $\begin{array}{l}\text { Senior } \\
\text { Leader }\end{array}$
\end{tabular}

level

CAC Agency

\begin{abstract}
Agency Level
\end{abstract}
Questionnaire

Information

specific to the

CAC,

including

annual

numbers of

forensic

interviews,

funding

structure, etc.

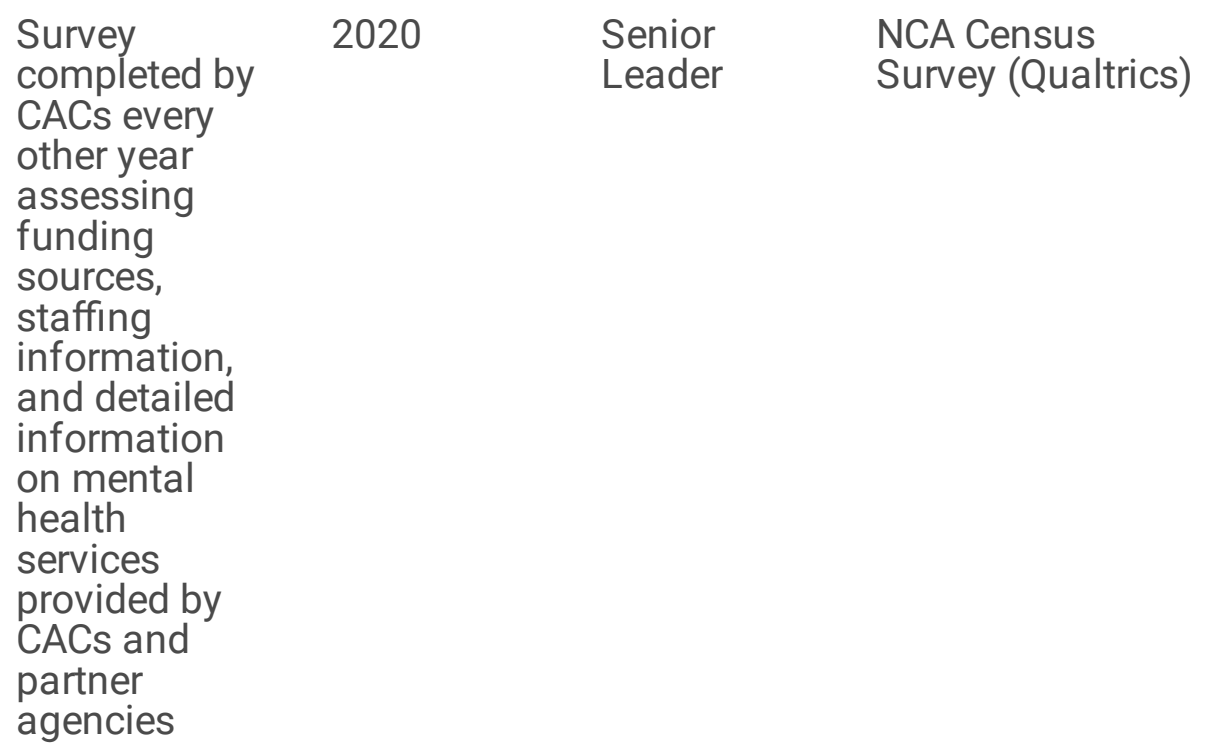

Survey completed by

CACs every

other year

assessing

funding

sources,

staffing

information,

and detailed

information

on mental

health

services

provided by

CACs and

partner

agencies

Senior

Leader

NCA Census

Survey (Qualtrics)

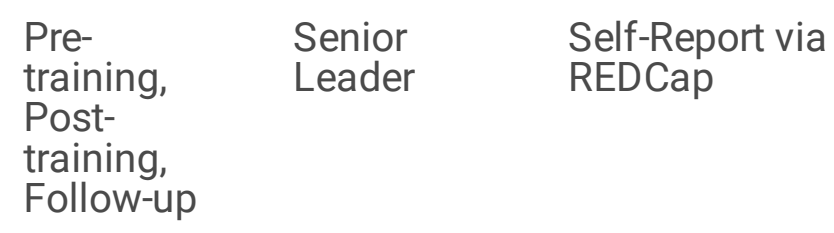

Pre- Senior

training, Leader

Self-Report via

Post-

training,

Follow-up

Leader

$\begin{array}{ll}\text { Multidisciplinary } & \begin{array}{l}\text { OMS MDT } \\ \text { Biannual } \\ \text { Survey }\end{array}\end{array}$

team

\author{
Anonymous, Twice \\ voluntary yearly \\ survey survey \\ completed by \\ members of \\ the MDT \\ regarding the \\ functioning of \\ MDT, \\ feedback on \\ the process, \\ and \\ suggestions
}


for improving

the MDT

Outcomes

CAC Outcome

Data

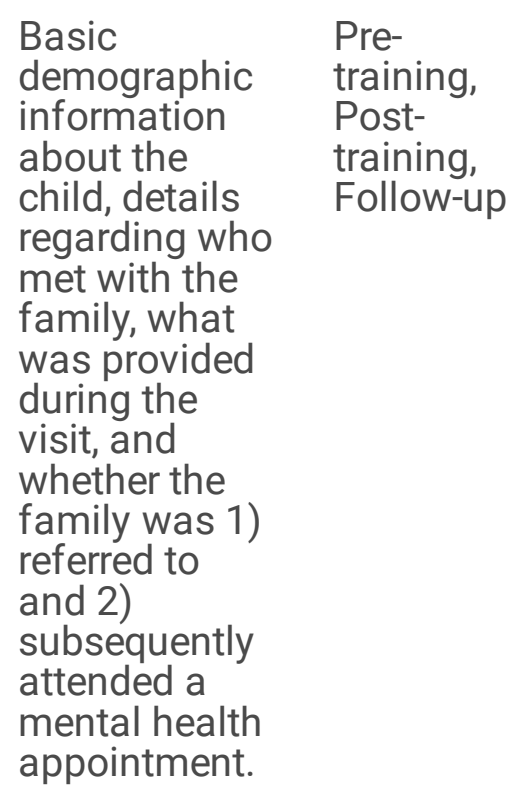

Basic

demographic information about the child, details regarding who met with the family, what was provided during the visit, and whether the family was 1) referred to and 2) subsequently attended a mental health appointment.

Post-

training,

Follow-up

Child Contact

Data

OMS Initial

Caregiver Survey
Anonymous, voluntary survey typically collected onsite at the end of the CAC visit via paper surveys or electronically; obtains family feedback regarding their visit and the support and follow-up provided by CAC staff
OMS Followup Caregiver Survey
Anonymous, voluntary survey typically completed via a phone call 4 to 8 weeks post visit; collects additional feedback regarding onsite visit, support, and subsequent

$\begin{array}{lll}\text { Pre- } & \text { Victim } & \text { REDCap } \\ \text { training, } & \text { Advocate } & \end{array}$

OMS Initial

Caregiver Survey $\begin{array}{ll}\text { Throughout } & \text { Caregiver } \\ \text { year, as per } & \text { report }\end{array}$

CAC

protocol 
follow-up by

CAC and MDT

staff

Screening

\begin{tabular}{|c|c|c|c|c|c|}
\hline $\begin{array}{l}\text { Mental Health } \\
\text { Screening }\end{array}$ & $\begin{array}{l}\text { Child } \\
\text { Behavioral } \\
\text { Health } \\
\text { Screener }\end{array}$ & $\begin{array}{l}\text { Pediatric } \\
\text { Symptom } \\
\text { Checklist } \\
\text { (PSC-17; [86]) } \\
\text { plus } 3 \text { items } \\
\text { focused on } \\
\text { clinical-level } \\
\text { child trauma } \\
\text { symptoms, } 6 \\
\text { items } \\
\text { assessing } \\
\text { functional } \\
\text { impairment, } \\
\text { and } 3 \text { critical } \\
\text { items } \\
\text { assessing } \\
\text { suicidality, } \\
\text { problematic } \\
\text { sexual } \\
\text { behavior, and } \\
\text { substance } \\
\text { use. }\end{array}$ & $\begin{array}{l}\text { Pre- } \\
\text { training, } \\
\text { Post- } \\
\text { training, } \\
\text { Follow-up }\end{array}$ & $\begin{array}{l}\text { Caregiver } \\
\text { report (total } \\
\text { score) }\end{array}$ & REDCap \\
\hline
\end{tabular}

Figures 


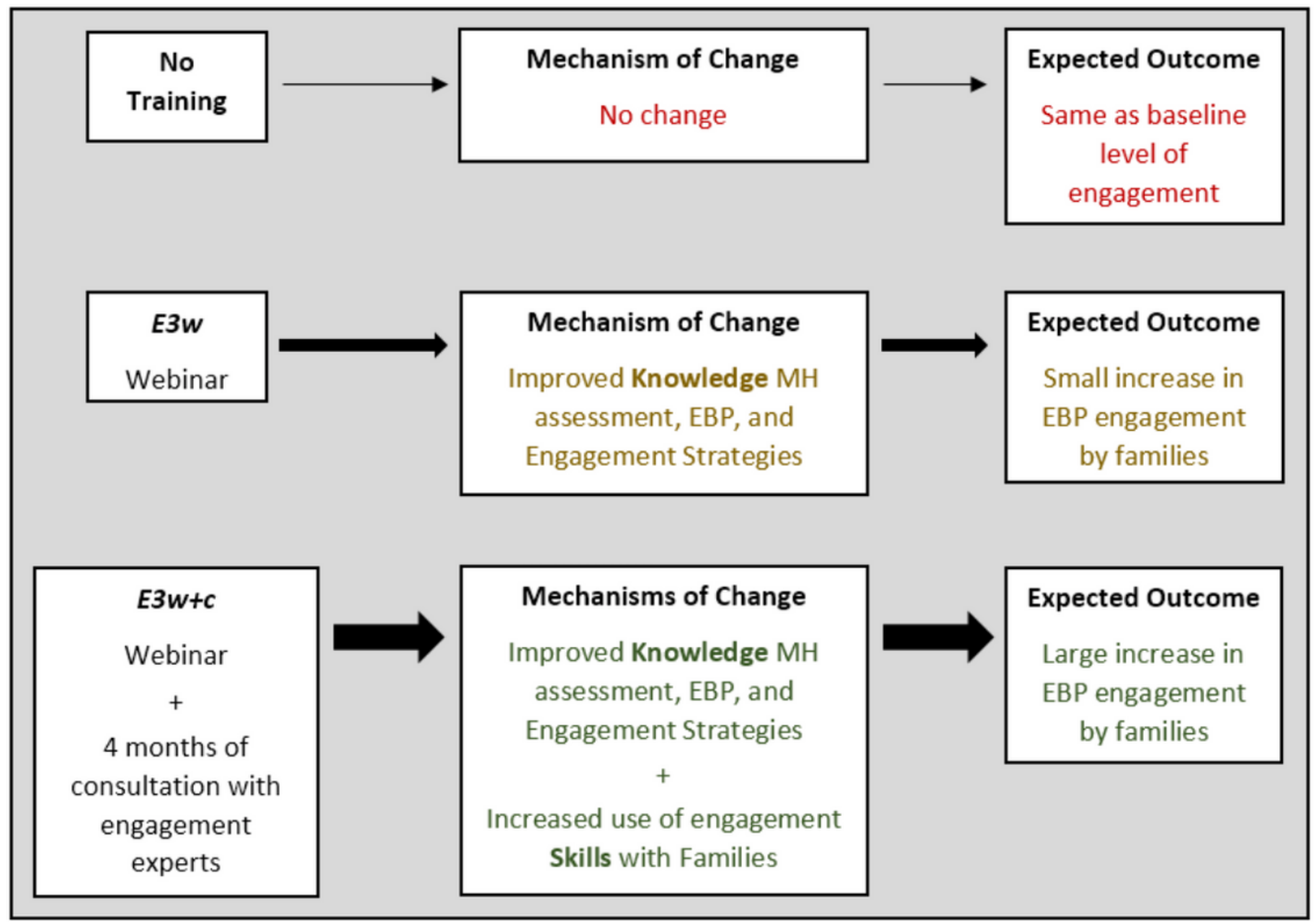

Figure 1

Flow chart of study premise and hypotheses. 


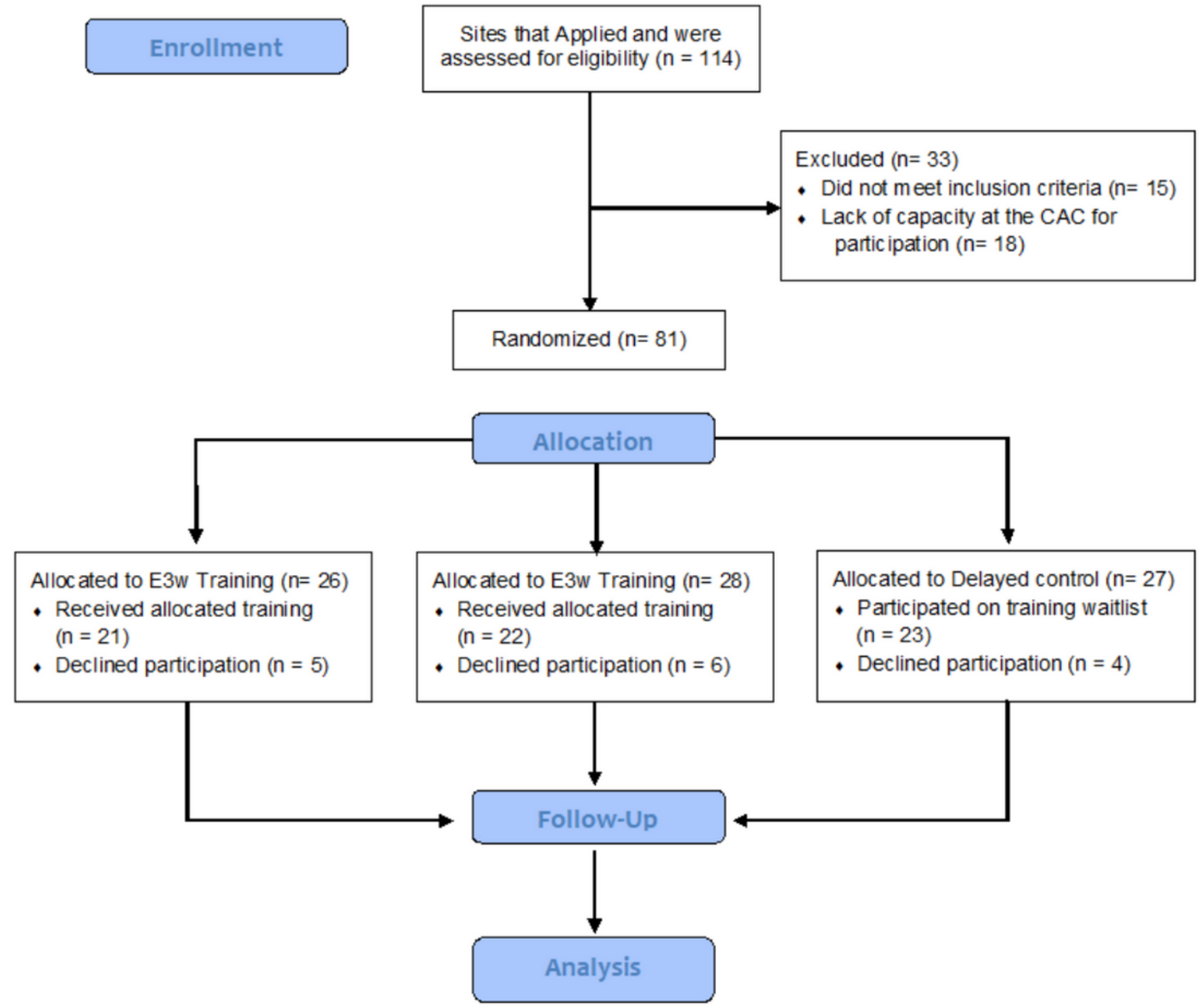

Figure 2

Flow diagram of site enrollment and randomization. 


\begin{tabular}{|c|c|c|c|c|c|}
\hline \multicolumn{6}{|c|}{ Data Collection Time Points } \\
\hline $\begin{array}{c}\text { December, 2019 } \\
\text { (Pre Training) }\end{array}$ & $\begin{array}{c}\text { January, 2020 } \\
\text { (Pre Training) }\end{array}$ & $\begin{array}{c}\text { Between January and June } \\
\text { (During Training) }\end{array}$ & \multicolumn{2}{c|}{$\begin{array}{c}\text { July, 2020 } \\
\text { (Post-Training) }\end{array}$} & $\begin{array}{c}\text { November, 2020 } \\
\text { (Follow-Up) }\end{array}$ \\
\hline \multicolumn{5}{|c|}{$\leftarrow$ OMS Caregiver Surveys $\rightarrow$} \\
\hline OMS MDT Survey & & & OMS MDT Survey & OMS MDT Survey \\
\hline Pre-training Survey & & & Child Contact Data + CBHS & Child Contact Data + CBHS \\
\hline & Child Contact Data + CBHS & & Engagement & Engagement \\
\hline & Engagement (Fidelity) & & & NCA Member Census \\
\hline
\end{tabular}

\section{Figure 3}

Data collection time points.

\section{Supplementary Files}

This is a list of supplementary files associated with this preprint. Click to download.

- SPIRITchecklistNavigatorProtocol.doc 